\title{
Multifeature Fusion Human Motion Behavior Recognition Algorithm Using Deep Reinforcement Learning
}

\author{
Chengkun Lu (iD) \\ College of Sports Science, Harbin Normal University, Harbin 150025, China \\ Correspondence should be addressed to Chengkun Lu; luchengkun@hrbnu.edu.cn
}

Received 13 August 2021; Revised 11 September 2021; Accepted 21 October 2021; Published 9 November 2021

Academic Editor: Jinan Fiaidhi

Copyright (c) 2021 Chengkun Lu. This is an open access article distributed under the Creative Commons Attribution License, which permits unrestricted use, distribution, and reproduction in any medium, provided the original work is properly cited.

\begin{abstract}
Through the recognition and analysis of human motion information, the actual motion state of human body can be obtained. However, the multifeature fusion of human behavior has limitations in recognition accuracy and robustness. Combined with deep reinforcement learning, multifeature fusion human behavior recognition is studied and we proposed a multifeature fusion human behavior recognition algorithm using deep reinforcement learning. Firstly, several typical human behavior data sets are selected as the research data in the benchmark data set. In the selected data sets, the behavior category contained in each video is the same behavior, and there are category tags. Secondly, the attention model is constructed. In the deep reinforcement learning network, the small sampling area is used as the model input. Finally, the corresponding position of the next visual area is estimated according to the time series information obtained after the input. The human behavior recognition algorithm based on deep reinforcement learning multifeature fusion is completed. The results show that the average accuracy of multifeature fusion of the algorithm is about $95 \%$, the human behavior recognition effect is good, the identification accuracy rate is as high as about $98 \%$ and passed the camera movement impact performance test and the algorithm robustness, and the average time consumption of the algorithm is only $12.7 \mathrm{~s}$, which shows that the algorithm has very broad application prospects.
\end{abstract}

\section{Introduction}

Human action recognition refers to the recognition and use of human behavior patterns and action categories $[1,2]$. Through the recognition and analysis of human motion information, the actual motion state of the human body can be obtained, and a variety of related services can be provided in combination with its actual needs. Some scholars abroad have divided human behavior into three levels: motor unit, action, and activity. The movement unit refers to the actual movement element composition of the movement [3]. Action refers to the combination of action elements according to a certain order to form an action sequence of a certain type of movement $[4,5]$. Activity refers to a broader concept, representing the complex movement of the human body, which is closely related to the object and the surrounding environment [6]. Recognition of human behavior is usually combined with multifeature fusion, and its application prospects are very broad. It has been extensively developed in many fields, including intelligent prosthetics, elderly monitoring, and motion detection $[7,8]$. For smart prostheses, the recognition of human behavior can help obtain the phase of a person's gait and formulate motor control strategies based on different phases to help the human body move. Regarding the monitoring of the elderly, the recognition of human behavior can help to detect and issue alarms in real time for various dangerous behaviors such as falls and monitor various actions of the elderly $[9,10]$. For exercise detection, the recognition of human behavior can help to calculate the calories consumed by exercise behavior based on the duration of different behaviors and exercise intensity data [11], so as to achieve the purpose of real-time monitoring of human exercise [12].

Literature [13] proposed deep reinforcement learning based on fuzzy reasoning to recognize the control behavior of intelligent traffic lights. Intelligent transportation systems overcome the limitations of traditional transportation systems and have become an important part of smart cities and have 
been widely used. In order to improve the efficiency of the traffic light control system, a dynamic intelligent traffic light control system that takes real-time traffic information as input and dynamically adjusts the duration of the traffic light is proposed. In addition, the proposed Dynamic and Intelligent Traffic Light Control System (DITLCS) operates in three modes: fair mode, priority mode, and emergency mode. Among them, all vehicles are regarded as equal priority, different types of vehicles are given different priority levels, and emergency vehicles are given the highest priority, respectively. Literature [14] proposed the study of the local optical flow method based on You Only Look Once (YOLO) in human action recognition. By calculating the optical flow modulus of the area where the human target is located, the amount of calculation is reduced, and the calculation time is saved. Then a threshold is set to complete the person's behavioral identity. Through the steps of design algorithm and experimental verification, the walking, running, and falling states of the human body in the indoor sports video in real life were identified. Experimental results show that the algorithm is more beneficial to the recognition of jogging behavior. Literature [15] proposed human behavior recognition in multiview videos, using deep learning techniques, including convolutional neural networks and long-short-term memory networks. A deep network is constructed under a multiviewpoint framework to learn the long-term relevance of human behavior recognition from videos. The use of two cameras as sensors effectively overcomes the problems of occlusion and contour blur and improves the accuracy of the multiview frame. Literature [16] proposed the application of the Principal Component Analysis-Long-Short-Term Memory (PCA-LSTM) model in human behavior recognition, collecting and processing the surface electromyogram (EMG) signals of the human upper limbs, and putting them into the PCA model for data dimensionality reduction. In addition, the dimensionality-reduced data is put into the Long ShortTerm Memory (LSTM) neural network model to classify human behavior, thereby calculating the classification efficiency and recognition rate.

The above methods have certain limitations in recognition accuracy and robustness. Deep reinforcement learning is combined to study the multifeature fusion human action recognition problem, and a multifeature fusion human action recognition algorithm based on deep reinforcement learning is proposed. The main contributions of the algorithm in this paper are as follows:

(1) This algorithm can realize the recognition of various human behaviors and has achieved good recognition results.

(2) The paper constructs a reinforcement learning model and proposes a specific structure of the deep reinforcement learning network recognition model

(3) This algorithm can quickly lock the character area after observation, the learning ability is more remarkable, and the overall robustness is strong.

(4) Several indexes are used to verify the effectiveness of the proposed algorithm.

\section{Related Works}

Regarding the problem of multifeature fusion human action recognition, related research has been carried out abroad for a long time, and various recognition algorithms of noncontact type and contact type have been proposed $[17,18]$. Among them, the noncontact type algorithms include the multifeature fusion human action recognition algorithm based on visual inspection technology. The contact type algorithms include the multifeature fusion human action recognition algorithm based on sensor detection and so on. Although domestic research on this issue started relatively late, breakthrough research results have been obtained in recent years, mainly for the recognition of a variety of human behaviors. Literature [19] proposed realizing the level control of human behavior through deep reinforcement learning, obtaining effective representation of the environment from high-dimensional sensory input, and using these to extend previous experience to new situations. Deep Q network is a new artificial intelligence trained by deep neural network. It can use end-to-end reinforcement learning to learn successful strategies directly from highdimensional sensory input. Literature [20] proposed the use of multiscale deep reinforcement learning to establish a large-scale quantitative image database and integrate the preliminary experience of human behavior analysis. In addition, the paper also explores the feasibility of a full-body volume analysis full-automatic workflow based on deep reinforcement learning as well as the influence of contrast and slice thickness on the calculation of organ volume. Multiscale DRL is used to detect the three-dimensional anatomical landmark points of the whole-body organ volume and three-dimensional organ segmentation. But the accuracy is not high.

Literature [21] proposed a human behavior recognition algorithm based on the fusion of image multiple features and conditional random field. The algorithm is composed of three basic cascade modules. Firstly, a cyclic neural network is constructed, and then feature similarity is introduced to form a more comprehensive and accurate use of feature similarity. Finally, the human behavior of image is recognized through conditional random field by using multiple features, and the algorithm has poor processing effect for multiple features. Literature [22] proposed a recognition algorithm based on multifeature fusion. In this algorithm, the change of local binary pattern feature distribution is related to projection error. For quick and accurate detection, the research data are extracted from professional facial expression database. The comparison shows the efficiency of this algorithm, but the recognition time is long. Literature [23] proposed a recursive neural network technology, which uses RGB and skeleton sequence for human activity recognition. The recognition accuracy needs to be further improved. Literature [24] proposed a driving assistant algorithm based on multifeature fusion, establishes a multifeature fusion model from the perspective of infrared image, establishes a spatiotemporal correlation model according to fuzzy set theory, and comprehensively analyzes and realizes the algorithm research, but it takes a long time. Literature 
[25] constructs a deep learning network structure, extracts and combines shallow and deep features, and uses neural network for feature weighted fusion to realize human behavior recognition, but the algorithm design is complex and time-consuming. Literature [26] constructs a multiview human motion map, extracts the gradient histogram of the image, uses the fusion algorithm to generate the feature vector, and further carries out the image feature separation to complete human behavior recognition, but the accuracy is not high.

\section{Human Action Recognition Algorithm Based on Multifeature Fusion Combined with Deep Reinforcement Learning}

3.1. Construction of Human Behavior Data. Several typical human behavior data sets are selected as research data from the benchmark data sets released by multiple human behavior research institutions. First, perform cluster analysis on the research data, using the K-means algorithm. The specific clustering criterion function is as follows:

$$
J=\sum_{i=1}^{k} \sum_{j=1}^{n}\left\|x_{j}^{(i)}-c_{i}\right\|^{2},
$$

where $x_{j}^{(i)} \in A_{i} ; c_{i}$ is the center of cluster $A_{i} ; k$ is the number of clusters, and $n$ is the size of data.

In this algorithm, the samples are allocated according to the minimum distance principle, which is as follows:

$$
D_{i}=\min \left\{\left|x-c_{i}\right|\right\} \text {, }
$$

where $D_{i}$ refers to the minimum distance, $x$ refers to the concentrated sample, and the specific formula is as follows:

$$
x \in\left(O_{1}, O_{2}, \ldots, O_{i}, \ldots, O_{n}\right),
$$

where $O_{n}$ refers to the $m$ sample.

Through the algorithm, the final output clustering is as follows:

$$
A=A_{1}, A_{2}, \ldots, A_{i}, \ldots, A_{k},
$$

where $A_{k}$ refers to the $k$ output cluster.

In the research data, the behavior categories contained in each video in the selected data set are the same behavior and have category tags. The data set is divided into a test subset and a training subset, and there is no intersection between the two.

For role-playing data sets with fixed scenes, the test subset and the training subset must contain different roles. Among them, the training subset is mainly used in parameter training. After the training is completed, the test subset is used to test the model, and the model parameters are not adjusted during the test, as shown in Table 1.

$\mathrm{KTH}$ is a total of four controlled scenes, and twenty-five people make six actions: clapping, waving, boxing, running, jogging, and walking. In the original video, samples are obtained by downsampling, and the sample pixels are $160 \times 120$. The camera is fixed when the sample is obtained, the background is single, and there are changes in lighting, appearance, and clothing, as well as changes in the scale of people.

There are nine types of actions included in Weizmann, which are swinging arms to take off, single jump, twohanded swing, one-handed swing, moving to one side, flying jump, jumping, running, and walking. All actions are singleplayer actions, keeping the background still. There are category markers and corresponding silhouette information of the human body in the data set.

There are fourteen types of actions included in IXMAS. Eleven people complete these fourteen types of actions. Each person repeats the same actions three times, fixes the camera, and keeps the light changes small. The video was shot at five angles, one top view direction and four side view directions [27]. Due to the large shooting angles, the visual difference of the same kind of action in the data set is relatively large, and thus it is relatively difficult to recognize.

There are two data sets in Hollywood; The first one was shot in a controlled environment, and the amount of video data is relatively small. The second is to extract action clips from Hollywood movies, which contain ten different scenes and twelve action categories. In the extracted video, the characters' poses, clothes, and expressions are quite different, there are interfering factors such as occlusion, light changes, the overall scene changes greatly, and the camera is also moving. Since the sample scene is close to the real scene, it is very challenging to recognize human behavior [28].

There are 51 behavior categories in HMDB51, including drinking, climbing, golfing, and horse riding. In the data set, there are videos with larger category differences and videos with smaller category differences. There are both videos with significant operating characteristics and larger operating ranges and videos with insufficient operating characteristics and smaller operating ranges [29]. Since this data set is collected on the Internet, the scale changes and the appearance of the characters varies greatly, and the background is also more complex, which is extremely challenging. Examples of samples in this data set are shown in Figure 1.

UCF101 mainly collects 101 human behaviors on the YouTube website. In general, these 101 human behaviors can be classified into five categories: playing musical instruments, human actions [30], interacting with objects, interacting with people, and sports. Examples of samples in this data set are shown in Figure 2.

3.2. Construct an Attention Model. In order to solve the selection problem of visual regions and time series, the attention model is constructed; that is, in the deep reinforcement learning network, a small sampling area is used as the model input, and the next vision is based on the time series information obtained after the input [31, 32]. The corresponding location of the area is estimated. First, build a reinforcement learning model, which mainly describes the reinforcement learning task through Markov decisionmaking: the intelligent body continuously interacts with the external environment to obtain environmental information. 
TABLE 1: Human behavior data set.

\begin{tabular}{lccccc}
\hline No. & Public time & Data set & Number of video segments & Number of categories & Mark \\
\hline 1 & 2004 & KTH & 392 & 6 & Fixed scene and role-play \\
2 & 2005 & Weizmann & 82 & 9 & Fixed scene and role-play \\
3 & 2006 & IXMAS & 111 & 11 & Fixed scene and role-play \\
4 & 2009 & Hollywood & 3670 & 51 & Movie Video (Hollywood) \\
5 & 2011 & HMDB51 & 6767 & 101 & Tubing video \\
6 & 2013 & UCF101 & 13330 & Tubing video \\
\hline
\end{tabular}

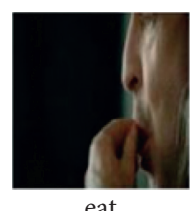

eat

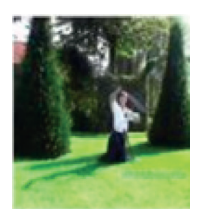

draw

sword

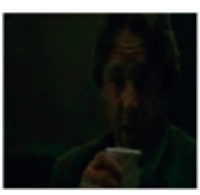

drink

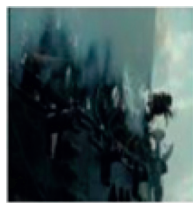

dive

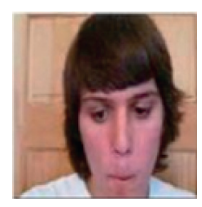

chew

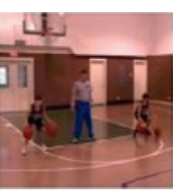

dribble

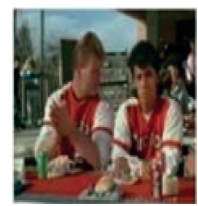

clap

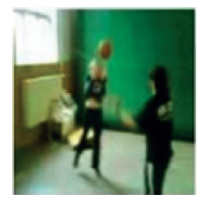

catch

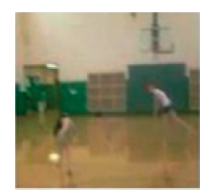

cartwheel

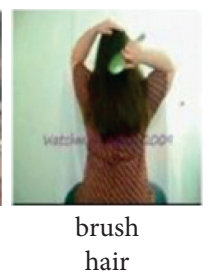

hair
Figure 1: Samples in HMDB51 data set.

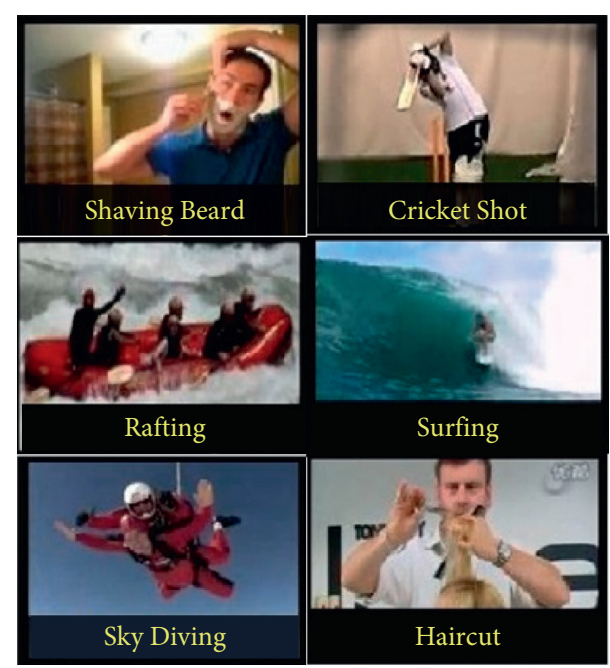

Figure 2: Samples in UCF101 data set.

The environmental information is the environmental state, which is the perceptual environment described by the agent. The agent decides the next action based on the obtained environmental information and influences the environment through the actions taken. First, a reinforcement learning model is built, as shown in Figure 3.

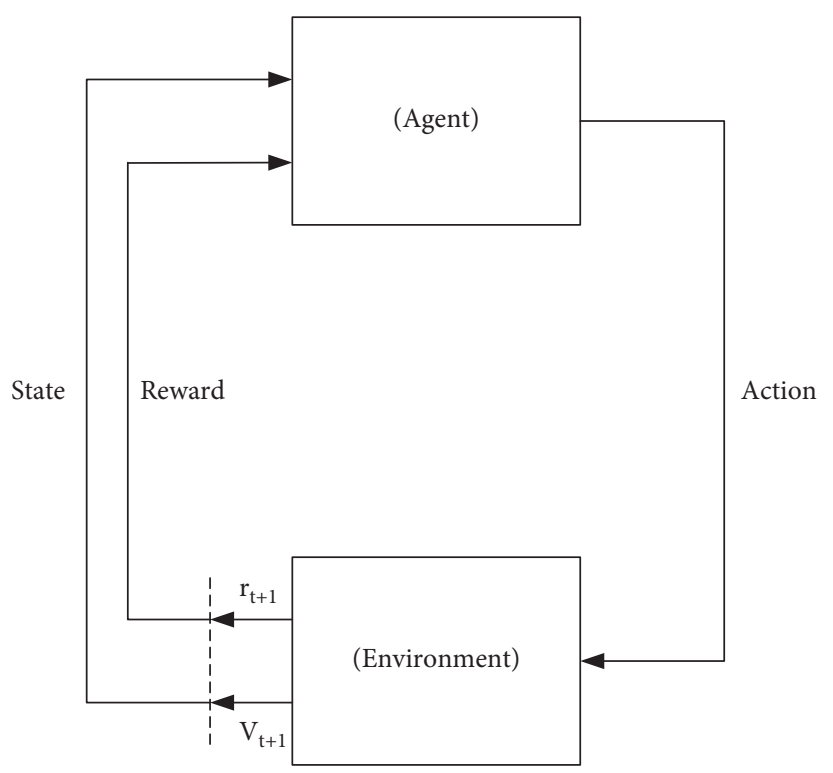

FIgURE 3: Reinforcement learning model constructed.

In the reinforcement learning model constructed in Figure 3, the agent is the core of the model. The agent continuously interacts with the external environment to obtain environmental information, which is the state of the environment (State), and at the same time obtains the enhanced information provided by the environment (Reward). The agent will decide the next action (Action) based on the perception information and influence the environment through the action taken.

The attention mechanism is realized through the constructed reinforcement learning model, where the environment is the input video sequence, denoted as $V$, the category is denoted as $y$, and the framework input at step $t$ is denoted as $v_{t}$. The deep reinforcement learning network is used as an agent [33].

First the reward function is defined as follows:

$$
R= \begin{cases}1, & y=\operatorname{argmax}_{y}\left(p_{T}\right), \\ 0, & y \neq \operatorname{argmax}_{y}\left(p_{T}\right),\end{cases}
$$

where $R$ refers to the reward function and $p_{T}$ refers to the times of enhancement.

Then the target function is defined. It is shown in the following formula: 


$$
J(\theta)=\sum_{S} \pi(S, \theta) R,
$$

where $J(\theta)$ refers to the target function, $S$ refers to the interaction between the environment and the intelligent body, and $\theta$ refers to the strategy parameter.

By gradient descent [34], the dynamics of strategy parameter $\theta$ are updated:

$$
\theta_{\text {new }}=\theta_{\text {old }}+\lambda \nabla_{\theta} J(\theta)
$$

where $\theta_{\text {new }}$ refers to the strategy parameter with the update dynamics; $\theta_{\text {old }}$ refers to the strategy parameter before the dynamics are updated; $\lambda$ refers to the dynamically updated threshold; $\nabla_{\theta}$ refers to the improved threshold.

After updating, the actual gradient of the objective function is as follows:

$$
\begin{aligned}
\nabla_{\theta} J(\theta) & =\nabla_{\theta} \sum_{S} \pi(S, \theta) R=\sum_{S} \nabla_{\theta} \pi(S, \theta) R, \\
& =\sum_{S} \pi(S, \theta) \frac{\nabla_{\theta} \pi(S, \theta) R}{\pi(S, \theta)} \\
& =\sum_{S} \pi(S, \theta) R \nabla_{\theta} \log \pi(S, \theta) .
\end{aligned}
$$

In the actual solution, the approximate solution of the actual gradient is taken. It is shown in the following formula:

$$
\nabla_{\theta} J(\theta) \approx \frac{1}{M} \sum_{m=1}^{M} R^{m} \nabla_{\theta} \log \pi\left(S^{m}, \theta\right),
$$

where $M$ refers to the number of samples taken for mathematical expectation value estimate; $m$ refers to the expected threshold.

The strategy probability is expanded. It is shown in the following formula:

$$
\pi(S, \theta)=\Pi_{t=1}^{T} \pi\left(S_{t}, \theta\right),
$$

where $T$ refers to the sample length; $t$ refers to Monte Carlo threshold.

The logarithmic derivative of the strategy probability is as follows:

$$
\nabla_{\mu} \lg \pi(S, \theta)=\frac{\left(\mu^{\prime}-\mu\right)}{\sigma^{2}},
$$

where $\mu$ refers to the estimated value; $\mu^{\prime}$ refers to the randomly output value; $\sigma$ refers to Gaussian distribution.

The strategy probability of data expansion obtains the expression of the gradient of the final objective function:

$$
\nabla_{\theta} J(\theta) \approx \frac{1}{M} \sum_{m=1}^{M} \sum_{t}^{T} R_{t}^{m} \nabla_{\theta} \log \pi\left(S_{t}^{m}, \theta\right) .
$$

Then the estimated reward function $b$ is introduced. To ensure the smoothness of the updated gradient [35], the updated gradient value is as follows:

$$
\nabla_{\theta} J(\theta) \approx \frac{1}{M} \sum_{m=1}^{M} \sum_{t}^{T}\left(R_{t}^{m}-b_{t}^{m}\right) \nabla_{\theta} \log \pi\left(S_{t}^{m}, \theta\right) .
$$

Finally, the updated gradient value is used as the attention mechanism.

\subsection{Build a Network Recognition Model for Deep Reinforcement}

Learning. Based on deep reinforcement learning, the deep reinforcement learning network recognition model is constructed, and the constructed model is mainly used for multifeature fusion human action recognition through 3D convolutional network. The specific structure of the model is shown in Figure 4.

In the construction of the deep reinforcement learning network recognition model, the human behavior image is first divided into $T$ units, and then, based on the deep reinforcement learning, the human and environment information is sensed and reinforced learning is performed on each unit in turn, combined with the 3D convolutional neural network method for feature calculation, as well as fusion of multiunit features, using the activation function (Softmax) to convert the feature value into a nonlinear feature, and finally the feature classification is completed by the mean value calculation.

\subsection{Human Action Recognition Algorithm Integrating Multiple} Features. The deep reinforcement learning algorithm is used to train the connection relationship between the hidden layer and the output layer, and the human action recognition algorithm is designed and integrated to realize the multifeature fusion human action recognition.

The hidden layer of algorithm refers to the deep integration of advanced information technology, giving full play to the characteristics of artificial intelligence technology and achieving autonomous decision-making, autonomous execution, and dynamic optimization in the identification process. The output layer of the algorithm refers to the deep mining of the data value in the recognition process to achieve auxiliary decision-making.

The fusion of human action recognition algorithm is described as follows:

Input: training samples and test samples of the deep reinforcement learning network recognition model

Output: recognized human behavior record organization

(1) In the multifeature fusion human action recognition, the operating environment of the artificial intelligence system uses a level 3 cloud platform to provide back-end services to securely control basic data and user information. All cloud platform servers provide on-site management and inspection of the computer room environment and equipment all the time.

(2) Registration and authentication are required for entry and exit, which will be provided by a dedicated person throughout the entire process. 


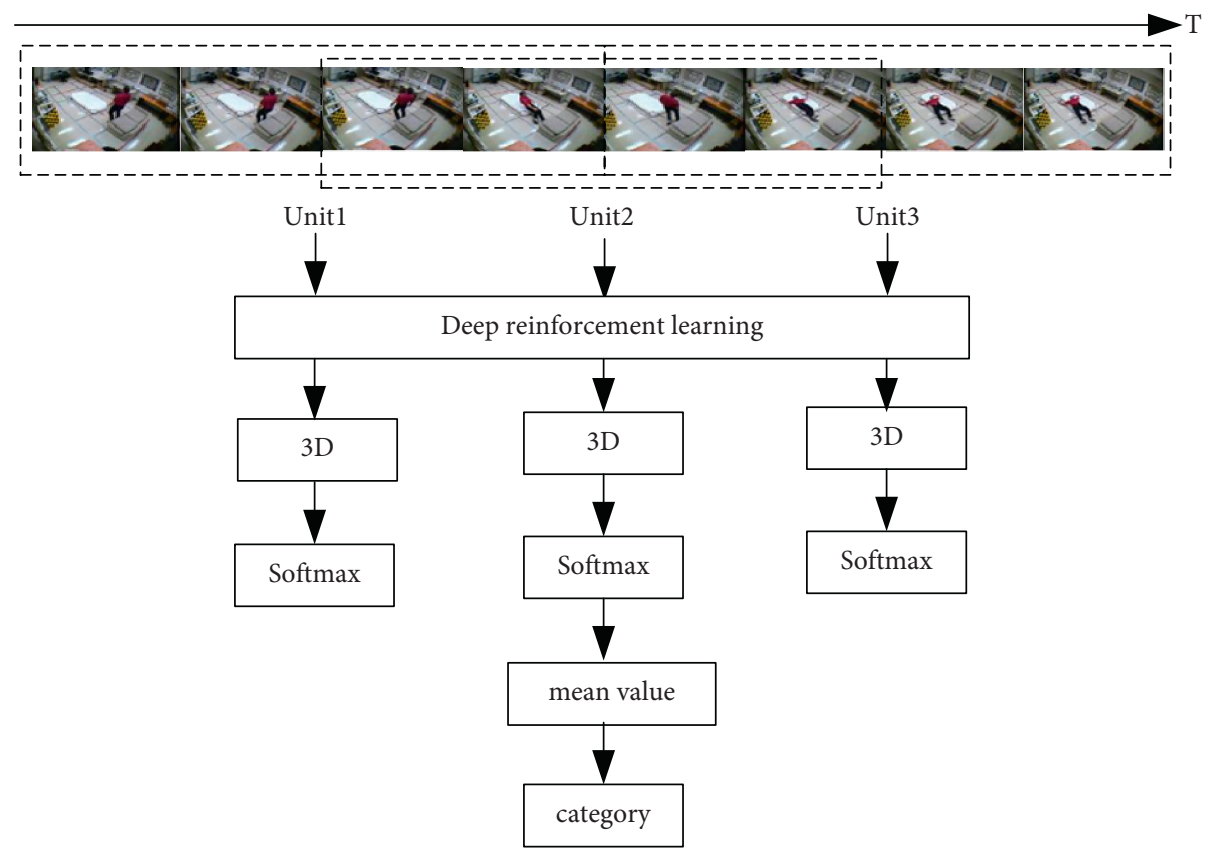

FIgURE 4: Specific structure of deep reinforcement learning network recognition model.

Network security and remote management of the cloud platform must be accessed through SSLVPN, different roles are assigned different access rights, and core device passwords are updated regularly. Public services only open specific services, applications, and ports.

(3) Based on application and port management and control, the firewall device is in a transparent mode, dedicated to data filtering.

(4) Port and address conversion is completed by a dedicated router.

(5) Suppose that $k_{f}^{\prime}$ refers to root tag tree generated from human behavior difference feature records, $f_{s}^{\prime}$ and $f_{w}^{\prime}$ are used to refer to node $i^{\prime}$ and node $j^{\prime}$ of the first-level subtree of the human action recognition feature tree, $R_{w}$ and $R_{k}$ are used to refer to the same denotation name, and then equation (14) is used to distinguish human behaviors with high similarity.

$$
h_{g}^{\prime}=k_{f}^{\prime}+s_{e} \frac{\left(f_{s}^{\prime}, f_{w}^{\prime}\right)}{\left(i^{\prime}, j^{\prime}\right)} \times\left(R_{w}, R_{k}\right) .
$$

(6) Suppose that $f_{\text {per }}$ refers to the content recognition of human behaviors; the calculation formula of $f_{\text {per }}^{\prime}$ is

$$
f_{\mathrm{per}}^{\prime}=\frac{h_{g}^{\prime}}{s_{e}}+p_{v} .
$$

In the above formula, $p_{v}$ refers to the tissue recognized in human body.

The operation flow of the fusion human behavior recognition algorithm is shown in Figure 5.

\section{Experimental Analysis and Results}

4.1. Experimental Environment and Data Set. Simulation experiments are performed on the designed multifeature fusion human action recognition algorithm based on deep reinforcement learning.

Running environment of simulation experiment is as follows: Windows 10 system, with CPU memory of $8 \mathrm{~GB}$. The main operating tool in the experimental environment is MAX + PLUSII, which provides an interface that can be used for other industry standard EDA tool software. This interface complies with EDIF200 and EDIF300 standards, LPM2.1 parametric module library, SDF2.0, VITAL95, Verilog HDL, VHDL1987, and VHDL1993 standards. Other EDA tools and software can be used to design input and then compiled and processed by MAX + PLUSII computer. Third-party EDA tools can also be used for equipment and board level simulation.

In the research data set, 88 videos are selected for model training in the experiment, and 37 videos are selected for model testing in the experiment. After completing the experimental parameter settings, the robustness and recognition accuracy of the multifeature fusion human action recognition algorithm based on deep reinforcement learning are tested, and the impact of camera movement on performance is also tested.

4.2. Experimental Steps. (a) Build simulation environment and debug operation parameters. (b) Collect human behavior data from six data sets: KTH, Weizmann, IXMAS, Hollywood, HMDB51, and UCF101, and preprocess the data as the data source. (c) The human behavior recognition effect of this algorithm is simulated, and other literatures are selected as comparison algorithms to simulate, and the 


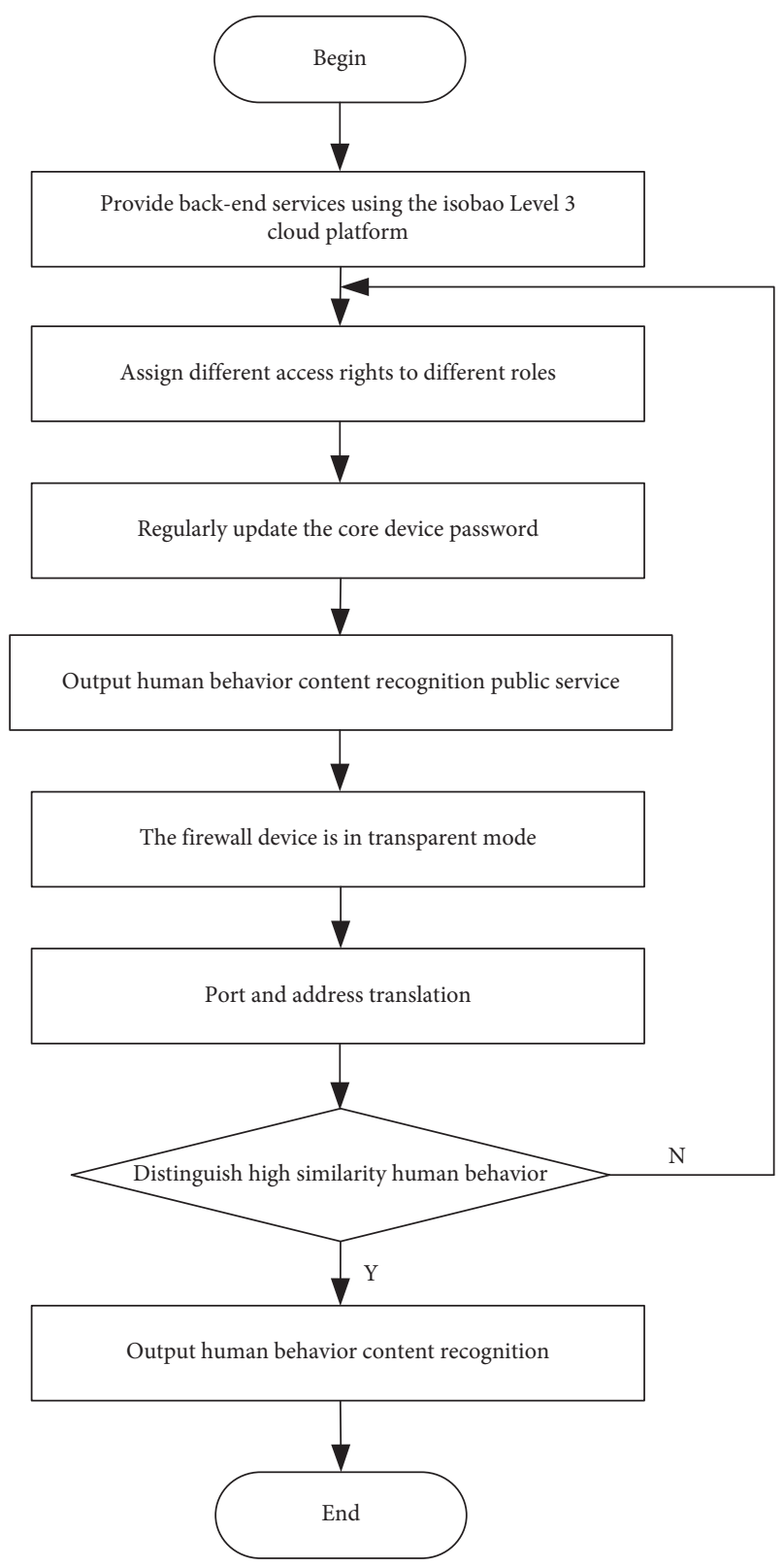

FIGURE 5: Operation process of fusion human behavior recognition algorithm.

recognition results of different algorithms are drawn. (d) Save the simulation results in fixed files to avoid repeated calculation. (e) Design a number of experimental indicators, test and analyze the algorithm on different data sets, and compare and verify the effect of human behavior recognition in this paper.

4.3. Experimental Standards. In the experiment, the parameter settings of the deep reinforcement learning network recognition model are shown in Table 2.

After setting the experimental parameters, the robustness and recognition accuracy of the multifeature fusion human behavior recognition algorithm based on deep
TABLE 2: Parameter settings of deep reinforcement learning network recognition model.

\begin{tabular}{lcc}
\hline No. & Parameter item & Value \\
\hline 1 & Number of hidden units & 512 \\
2 & Sequence length value & 10 \\
3 & Model initial parameter acquisition method & CNN \\
4 & Minimum group size & 32 \\
5 & Momentum factor & 0.9 \\
6 & Weight attenuation & $5 \times 10^{-4}$ \\
7 & Initial learning rate & $10^{-3}$ \\
8 & Number of training rounds & 128 \\
9 & Select frame policy superparameters & 10 \\
10 & Select image block policy superparameters & 70 \\
11 & Frame relative position & $1-10$ frames \\
12 & Frame sequence length & 10 \\
13 & Image block threshold & 70 pixels \\
\hline
\end{tabular}

reinforcement learning are tested, and the influence of camera movement on the performance is tested.

(1) The recognition accuracy of the algorithm: In order to enhance the comparison of the experimental results, the existing algorithms are used as the comparison test items for comparison experiments, and the experimental data of the recognition accuracy are also obtained and compared. The comparative experiment algorithm includes multifeature fusion human action recognition algorithm based on visual detection technology, sensor detection, and radio frequency electronic tag.

$$
S_{B G}=\frac{Z_{F J}}{G_{I I}} \times 100 \%
$$

In the above equation, $Z_{F J}$ refers to the number of samples correctly recognized, and $G_{I I}$ refers to the size of the entire test samples.

(2) Algorithm robustness: The robustness of the multifeature fusion human action recognition algorithm based on deep reinforcement learning is tested, including the situation where the initial sampling affects the performance and the situation where the camera movement affects the performance. For the test of the impact of initial sampling on performance, the selected observation window is of $76 \times 76$ pixels, and the initial observation window is randomly selected, or the character area is determined as the initial observation window.

(3) Camera movement affects performance: The performance of the camera movement is tested and the changes in the recognition rate after the camera movement is eliminated are observed.

(4) Recognition time consuming: The running time of the algorithm is one of the important indicators to reflect its performance. Therefore, in order to verify the efficiency of the algorithm in this paper, the recognition time is selected as the indicator. 

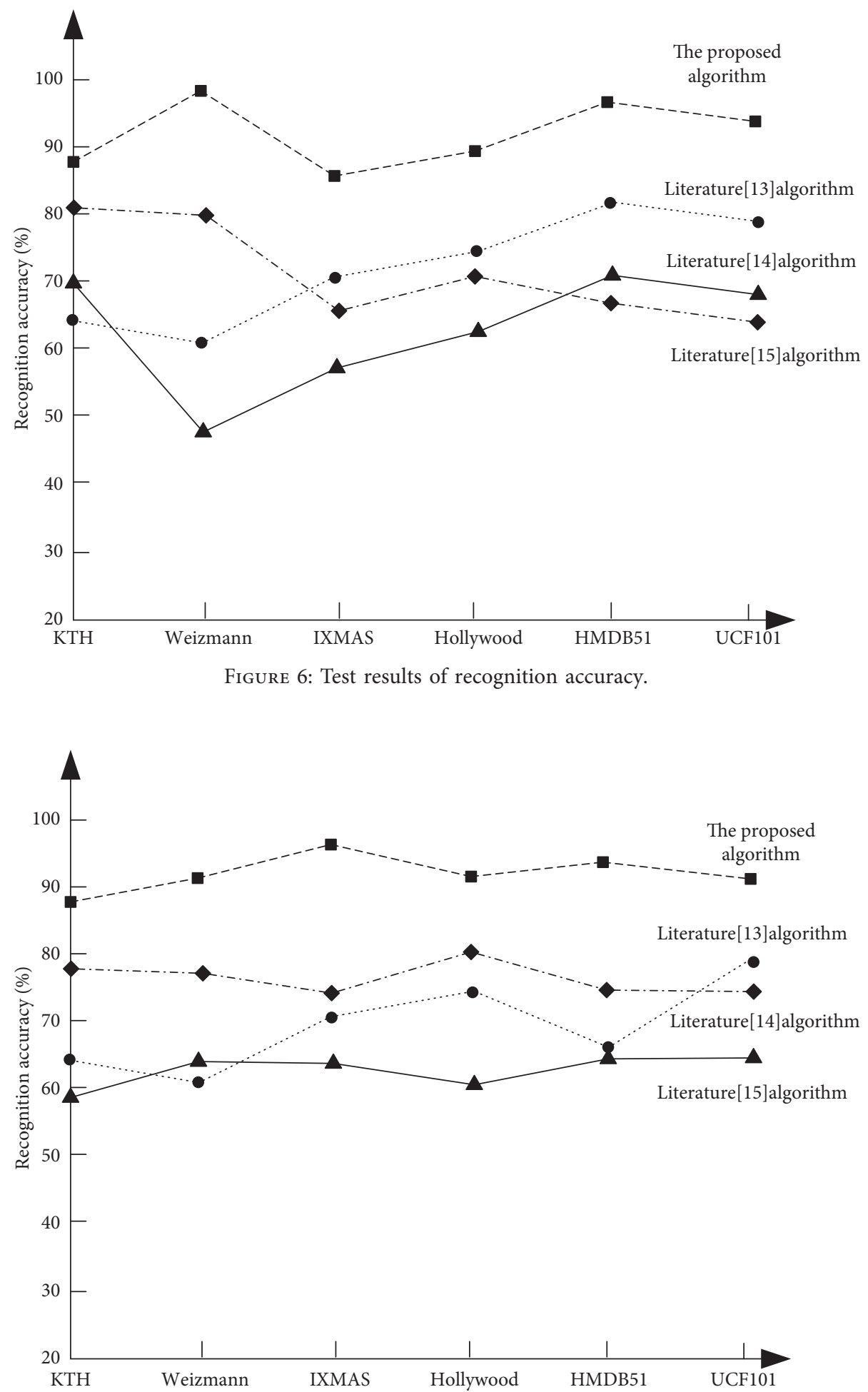

FIgURE 7: Test results of recognition accuracy.

\subsection{Results and Discussion}

4.4.1. Comparison of Recognition Accuracy Test. The test results of recognition accuracy are shown in Figures 6 and 7. Among them are the test results of the recognition accuracy of the first half of the videos in the experimental video data set.
The test results of the recognition accuracy of the second half of the video in the experimental video data set are shown in Figure 7.

The highest accuracy of the algorithms in literature [13], literature [14], and literature [15] is no more than $80 \%$, which is significantly lower than that in this paper. It can be seen that the deep reinforcement learning technology 
TABLE 3: Specific test results of initial sampling affecting performance.

\begin{tabular}{|c|c|c|}
\hline Randomly select the initial observation window & Data set & Recognition rate (\%) \\
\hline \multirow{13}{*}{ After determining the person area, take the person area as the initial observation window } & KTH & 92.65 \\
\hline & Weizmann & 96.32 \\
\hline & IXMAS & 92.38 \\
\hline & Hollywood & 95.65 \\
\hline & HMDB51 & 96.57 \\
\hline & UCF101 & 94.21 \\
\hline & Data set & Recognition rate $(\%)$ \\
\hline & KTH & 93.63 \\
\hline & Weizmann & 97.20 \\
\hline & IXMAS & 93.69 \\
\hline & Hollywood & 96.52 \\
\hline & HMDB51 & 97.32 \\
\hline & UCF101 & 95.21 \\
\hline
\end{tabular}

TABLE 4: Specific test results of camera movement affecting performance.

\begin{tabular}{lccc}
\hline No. & Data set & Recognition rate of camera movement not eliminated (\%) & Eliminate recognition rate after camera movement (\%) \\
\hline 1 & KTH & 92.30 & 93.52 \\
2 & Weizmann & 93.01 & 94.10 \\
3 & IXMAS & 94.20 & 95.33 \\
4 & Hollywood & 90.36 & 91.24 \\
5 & HMDB51 & 86.32 & 87.52 \\
6 & UCF101 & 84.20 & 85.32 \\
\hline
\end{tabular}

Table 5: Comparison of human behavior recognition time.

\begin{tabular}{lcccc}
\hline Data set & The proposed algorithm & Algorithm in literature [13] & Algorithm in literature [14] & Algorithm in literature [15] \\
\hline KTH & 12 & 30 & 42 & 25 \\
Weizmann & 13 & 36 & 39 & 26 \\
IXMAS & 12 & 39 & 36 & 39 \\
Hollywood & 10 & 33 & 35 & 35 \\
HMDB51 & 15 & 40 & 42 & 40 \\
UCF101 & 14 & 42 & 41 & 46 \\
Mean value & 12.7 & 36.7 & 39.2 & 35.2 \\
\hline
\end{tabular}

adopted in this paper has very efficient computational performance and can effectively improve the accuracy of human behavior recognition. In this paper, the highest recognition accuracy is about $98 \%$, and the recognition effect is better.

4.4.2. Comparison of Robustness Test. The specific test results of initial sampling affecting performance are shown in Table 3 .

According to Table 3, the recognition rate is higher after the character area is determined as the initial observation window, but the improvement effect is not significant. This shows that the algorithm can quickly lock the character area after observation, the learning ability is more remarkable, and the overall robustness is strong.

4.4.3. Comparison of Camera Movement Performance Test. When testing the impact of camera movement on performance, the initial observation window is randomly selected.
The specific test results of camera movement affecting performance are shown in Table 4.

According to Table 4, the recognition rate is slightly improved after eliminating camera movement, indicating that camera movement does not have a big impact on performance, and the overall performance of the algorithm is relatively strong.

\section{Comparison of Recognition Time Consumption}

The human behavior recognition time of different algorithms is tested on six different data sets. The results are shown in Table 5.

According to the time consumption comparison results of human behavior recognition of different algorithms in Table 5, it can be found that the average time consumption of the algorithm in literature [15] is the highest, reaching $49.3 \mathrm{~s}$, while the average time consumption difference of the algorithms in literature [13] and literature [14] is small, but all 
of them are more than $35 \mathrm{~s}$, while the average time consumption of the proposed algorithm is only $12.7 \mathrm{~s}$, indicating that the algorithm in this paper runs faster and is more efficient. It has certain advantages in practical application.

\section{Conclusions}

Combining deep reinforcement learning to study the problem of multifeature fusion human action recognition, a multifeature fusion human action recognition algorithm based on deep reinforcement learning was designed, which achieved the improvement of recognition accuracy and stable robustness. The results show that the recognition accuracy of the proposed algorithm is higher than those of four other algorithms. After determining the person area, the recognition rate is higher, and the robustness performance is stronger after the person area is used as the initial observation window. After eliminating camera movement, the recognition rate has been slightly improved, and it has good applicability.

Multifeature fusion human action recognition is still under development, and various types of recognition algorithms are gradually being proposed. It is necessary to continuously optimize human action recognition according to the development of deep reinforcement learning, so as to provide a basis for the integration of multifeature human action recognition truly and accurately. The data set used in the proposed algorithm is relatively old, and it will be updated in future research, and the recognition accuracy of the algorithm will be further improved. (1) In terms of feature recognition, the proposed algorithm does not involve selecting suitable predictive features in different projects. Therefore, in the follow-up research process, fully consider the different characteristics of the identification algorithm, and carry out a new measurement based on the actual development situation. (2) In the recognition process, the recognition accuracy is improved through deep reinforcement learning. However, from an experimental perspective, research should be carried out on the multifeature fusion human action recognition obtained after deep reinforcement learning to improve the prediction effect.

\section{Data Availability}

The data used to support the findings of this study are included within the article. Readers can access the data supporting the conclusions of the study from Weizmann, KTH, IXMAS, Hollywood, HMDB51, and UCF101 data sets.

\section{Conflicts of Interest}

The author declares that there are no conflicts of interest with any financial organizations regarding the material reported in this manuscript.

\section{Acknowledgments}

This work was supported by Research and Planning Project of Philosophy and Social Sciences in Heilongjiang Province (19TYC156).

\section{References}

[1] R. Sizyakin, V. Voronin, N. Gapon, A. Zelensky, and A. Pižurica, "A deep learning-based approach for defect detection and removing on archival photos," Electronic Imaging, vol. 12, no. 15, pp. 59-62, 2020.

[2] J. Yu, M. Tan, H. Zhang, D. Tao, and Y. Rui, "Hierarchical deep click feature prediction for fine-grained image recognition," IEEE Transactions on Pattern Analysis and Machine Intelligence, vol. 1, 2019.

[3] J. Yu, D. Tao, M. Wang, and Y. Rui, "Learning to rank using user clicks and visual features for image retrieval," IEEE Transactions on Cybernetics, vol. 45, no. 4, pp. 767-779, 2015.

[4] S. Boukil, M. Biniz, F. E. Adnani, L. Cherrat, and A. E. E. Moutaouakkil, "Arabic text classification using deep learning technics," International Journal of Grid and Distributed Computing, vol. 11, no. 9, pp. 103-114, 2018.

[5] J. P. D. Yoon and W.-H. Lee, "Architectural factors detection from plan by deep learning framework," International Journal of Grid and Distributed Computing, vol. 11, no. 1, pp. 57-64, 2018.

[6] J. W. Kim, B. J. Park, H. Yoo, T. H. Oh, J. H. Lee, and J. M. Lee, "A model-based deep reinforcement learning method applied to finite-horizon optimal control of nonlinear control-affine system," Journal of Process Control, vol. 87, no. 6, pp. 166-178, 2020.

[7] P. Srivastava, D. Agarwal, and A. Bansal, "Ear based human identification using a combination of wavelets and multi-scale local binary pattern," International Journal of Future Generation Communication and Networking, vol. 12, no. 3, pp. 41-56, 2019.

[8] R. R. Peesari, J. Fiaidhi, and S. Mohammed, "Business success through understanding human emotions: case study of classifying emotions using the brain waives EEG data," International Journal of Disaster Recovery and Business Continuity, vol. 10, pp. 7-18, 2019.

[9] Y.-K. Lee and D.-W. Park, "Design of Internet of things business model with deep learning artificial intelligence," International Journal of Grid and Distributed Computing, vol. 11, no. 7, pp. 11-22, 2018.

[10] T. F. Denson, B. J. W. Dixson, A. N. Tibubos, E. Zhang, E. Harmon-Jones, and M. M. Kasumovic, "Violent video game play, gender, and trait aggression influence subjective fighting ability, perceptions of men's toughness, and anger facial recognition," Computers in Human Behavior, vol. 104, no. 3, pp. 1-10, 2020.

[11] J. Jun Yu, Y. Dacheng Tao, and D. Tao, "Click prediction for web image reranking using multimodal sparse coding," IEEE Transactions on Image Processing, vol. 23, no. 5, pp. 2019-2032, 2014.

[12] Z. Xia, J. Xing, C. Wang, and X. Li, "Gesture recognition algorithm of human motion target based on deep neural network," Mobile Information Systems, vol. 2021, Article ID 2621691, 12 pages, 2021.

[13] N. Kumar, S. S. Rahman, and N. Dhakad, "Fuzzy inference enabled deep reinforcement learning-based traffic light control for intelligent transportation system," IEEE Transactions on Intelligent Transportation Systems, vol. 12, no. 99, pp. 1-10, 2020.

[14] H. Zheng, J. Liu, and M. Liao, "Study on local optical flow method based on YOLOv3 in human behavior recognition," Journal of Computer and Communications, vol. 09, no. 1, pp. 10-18, 2021. 
[15] Y.-L. Hsueh, W.-N. Lie, and G.-Y. Guo, "Human behavior recognition from multiview videos," Information Sciences, vol. 517, pp. 275-296, 2020.

[16] M. Qiao and H. Li, "Application of PCA-LSTM model in human behavior recognition," Journal of Physics: Conference Series, vol. 1650, no. 3, pp. 32-37, 2020.

[17] J.-J. Kim, S.-H. Cha, K.-H. Cho, and M. Ryu, "Deep reinforcement learning based multi-agent collaborated network for distributed stock trading," International Journal of Grid and Distributed Computing, vol. 11, no. 2, pp. 11-20, 2018.

[18] S. Khan, P. Matani, R. S. Siddiqui, R. Tahir, and S. S. Ashraf, "Classification of benign melanocytic skin lesion using ABCD features and convolutional neural network (CNN)," International Journal of Future Generation Communication and Networking, vol. 13, no. 4, pp. 23-32, 2020.

[19] M. Volodymyr, K. Koray, S. David et al., "Human-level control through deep reinforcement learning," Nature, vol. 518, no. 7540, pp. 529-533, 2019.

[20] D. J. Winkel, H.-C. Breit, T. J. Weikert, and B. Stieltjes, "Building large-scale quantitative imaging databases with multi-scale deep reinforcement learning: initial experience with whole-body organ volumetric analyses," Journal of Digital Imaging, vol. 34, no. 1, pp. 124-133, 2021.

[21] X. Song, H. Zhou, and G. Liu, "Human behavior recognition based on multi-feature fusion of image," Cluster Computing, vol. 22, no. 1, pp. 9113-9121, 2018.

[22] L. Mao, N. Wang, L. Wang, and Y. Chen, "Classroom microexpression recognition algorithms based on multi-feature fusion," IEEE Access, vol. 7, no. 99, pp. 64978-64983, 2019.

[23] P. Verma, A. Sah, and R. Srivastava, "Deep learning-based multi-modal approach using RGB and skeleton sequences for human activity recognition," Multimedia Systems, vol. 26, no. 6, pp. 671-685, 2020.

[24] A. Dz, Q. B. Shi, and S. C. Yang, "A driver-assistance algorithm based on multi-feature fusion," Infrared Physics \& Technology, vol. 116, no. 15, Article ID 103747, 2021.

[25] Y. Huang, C. Wan, and H. Feng, "Multi-feature fusion human behavior recognition algorithm based on convolutional neural network and short-term memory neural network," Progress in laser and optoelectronics, vol. 056, no. 7, pp. 235-241, 2019.

[26] T. Liu, Y. Li, and L. Zhang, "Human behavior recognition of multi view depth motion map," Chinese Journal of image and graphics, vol. 24, no. 3, pp. 80-89, 2019.

[27] J. Zhou, D. Zhang, and W. Zhang, "A Multifeature fusion method for the color distortion and low contrast of underwater images," Multimedia Tools and Applications, vol. 3, no. 25, pp. 1-27, 2021.

[28] G. V. D. L. Cruz, Y. Du, and M. E. Taylor, "Pre-training with non-expert human demonstration for deep reinforcement learning," The Knowledge Engineering Review, vol. 34, no. 5, pp. 25-32, 2019.

[29] Y. Wu, N. Yang, Z. Chen, and B. Hua, "Multi-feature fusion based relative pose adaptive estimation for on-orbit servicing of non-cooperative spacecraft," Journal of Harbin Institute of Technology, vol. 26, no. 6, pp. 23-34, 2019.

[30] F. Li, Y. Fan, X. Zhang et al., "Multi-feature fusion method based on EEG signal and its application in stroke classification," Journal of Medical Systems, vol. 44, no. 2, pp. 66-72, 2020.

[31] X. Li, J. Wei, and H. Jiao, "Real-time tracking algorithm for aerial vehicles using improved convolutional neural network and transfer learning," IEEE Transactions on Intelligent
Transportation Systems, vol. 2021, Article ID 3072872, 10 pages, 2021.

[32] M. A. Hasan, N. A. Abdullah, M. M. Rahman, M. Y. I. B. Idris, and O. F. Tawfiq, "Dental impression tray selection from maxillary arch images using multi-feature fusion and ensemble classifier," IEEE Access, vol. 12, no. 99, pp. 1-8, 2021.

[33] G. Lederman, M. N. Rabe, and S. A. Seshia, "Learning heuristics for automated reasoning through deep reinforcement learning," Environmental Pollution, vol. 12, no. 25, pp. 1-12, 2019.

[34] J. Qin, Y. Huang, and W. Wen, "Multi-scale feature fusion residual network for Single Image Super-Resolution," Neurocomputing, vol. 379, no. 28, pp. 334-342, 2020.

[35] L. T. Alemu and M. Pelillo, "Multi-feature fusion for image retrieval using constrained dominant sets," Image and Vision Computing, vol. 94, no. 262, pp. 103-110, 2020. 